\title{
HPV not just a young woman's problem
}

\author{
Cite as: CMAJ 2017 March 13;189:E416-7. doi: 10.1503/cmaj.1095398
}

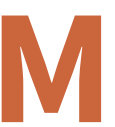
any Canadians may not be vaccinated against human papillomavirus (HPV) because their doctors hold outdated notions about the virus, said a women's reproductive health expert at a recent forum in Toronto hosted by the Society of Obstetricians and Gynaecologists of Canada.

"Most providers still think of this as a female thing," or a problem for "young promiscuous patients," said Dr. Nancy Durand, a gynecologist at Toronto's Sunnybrook Health Science Centre. But the latest evidence makes it clear, "we should discuss this with every single patient."

HPV is the most common sexually transmitted infection worldwide. Four out of five Canadians will get the virus at some point, though in most cases the infection clears without treatment within two years. But one in five times, the infection persists and may cause genital warts or cancers of the cervix, vagina, penis, anus, mouth or throat. Three vaccines provide more than
$90 \%$ protection against the strains of HPV that cause most of these cancers, but uptake remains patchy.

Part of the problem is that many doctors underestimate the prevalence of HPV infection or its risks, said Durand. "They still think cervical cancer and genital warts, because that's what we were taught."

While cervical cancers have decreased steadily since 1992, likely due to increased screening and earlier treatment of precancers, the incidence of other HPV cancers has recently surged, particularly among men.

According to Canadian Cancer Statistics 2016, mouth and throat cancers from HPV have increased $56 \%$ in men and $17 \%$ in women since the mid-1990s, and now make up a third of HPV cancers, tied with cervical cancer. Men are up to 4.5 times more likely to be diagnosed with these oropharyngeal cancers than women.

The rate of anal cancers from HPV has also increased by an average of $3 \%$ per

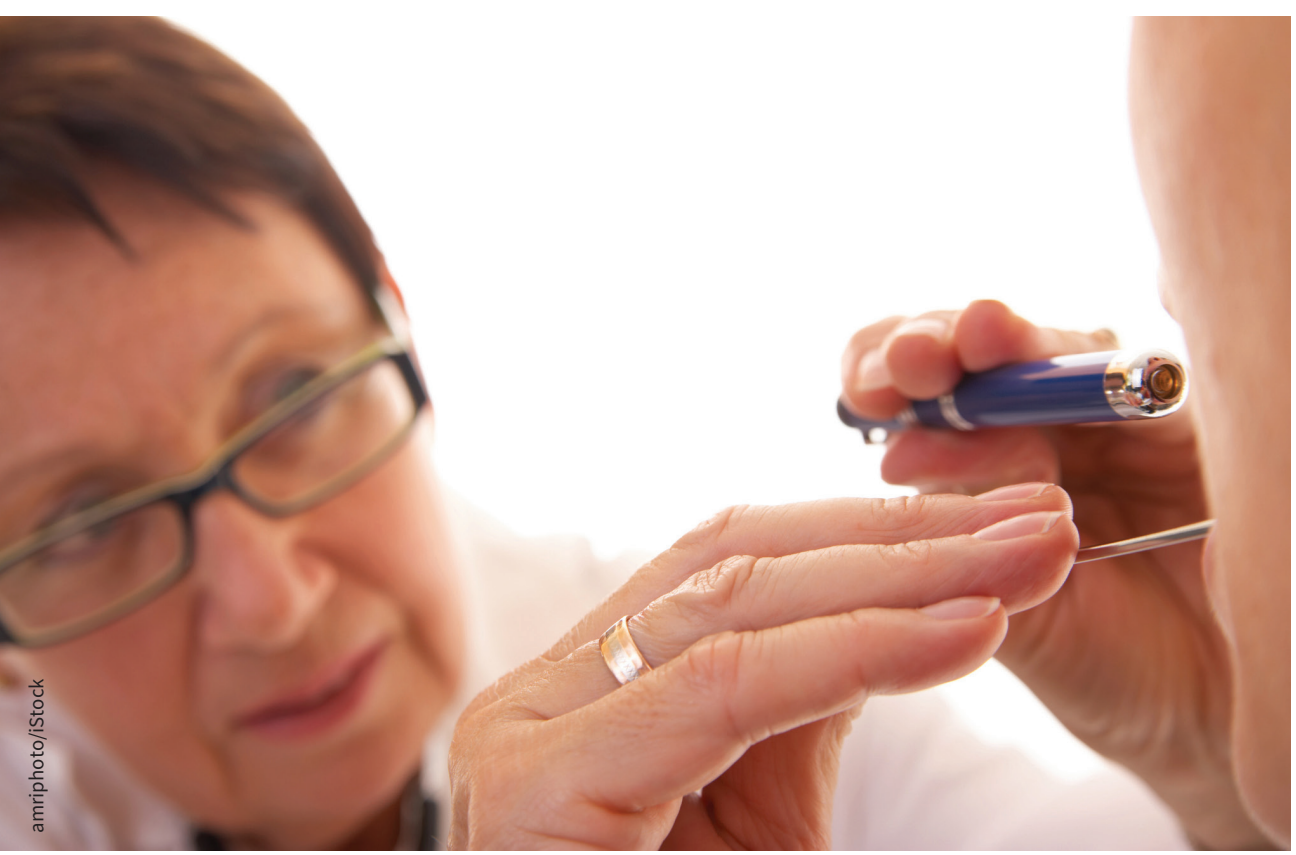

Some doctors still think of human papillomavirus as a young woman's problem, despite a surge in related oral cancers among men. year over the same period. Although traditionally these cancers hit gay men hardest, "the number of anal cancers in women is now surpassing men, including gay men," Durand said.

Vaccinating both men and women will be critical to curb these non-cervical cancers, because there's "no equivalent to cervical pap screening for these other areas," she said. Men in particular "fly under the radar."

There aren't good Canadian data, but an article published in JAMA Oncology in January showed that nearly half of American men have a genital HPV infection, and more than a quarter have at least one type of the virus known to cause cancer. "We're really just waiting for them to develop disease before we know that they have the virus," Durand said.

Last year, Ontario and Quebec followed the lead of Alberta, Nova Scotia, British Columbia and Prince Edward Island to expand school HPV vaccination programs to boys. National guidelines recommend both the four-strain and nine-strain HPV vaccines for Canadians over age nine with no upper age limit.

Some doctors may assume vaccination is only necessary for younger patients, because they're changing partners more often. But that doesn't mean the risk for older adults is zero, particularly when people are marrying later and more than a third of marriages end in divorce. "If you're over the age of 30 , your five-year risk of acquiring HPV is more than $20 \%$," said Durand. "Over the age of 45 , you still have more than a $10 \%$ risk."

Many people also assume that it's too late to be vaccinated if they already have HPV, or have had an infection in the past. But Durand noted that vaccination can reduce recurrence of cervical and anal precancerous cells, as well as genital warts. "Those of us who are specialists treating patients with HPV-related disease 
should not forget to counsel them about the value of vaccinating," she said. "It's not too late for age and it's not too late if you've already had disease in the past."

The strength of a health provider's recommendation is the most important factor in patients getting vaccinated, so medical organizations need to step up, continuing education programs about HPV, Durand said. "We do a lot of them, but I still feel like we haven't reached who we need to reach."

Just like with patients, repetition will be essential to getting the message across to clinicians that HPV vaccination is important to consider for all sexually active Canadians, Durand added. "When we hear it once, it just doesn't sink in, so we have to do these programs often."

Lauren Vogel, CMAJ 